\title{
LA LARGA DURACIÓN Y LA COYUNTURA. EL AÑO 2012 EN LAS RELACIONES VECINALES ENTRE CHILE, PERÚ Y BOLIVIA. RIESGOS Y, SOBRE TODO, OPORTUNIDADES
}

Fernando Wilson ${ }^{1}$

En la disciplina de la Historia ya es tradicional aludir a que sólo se la puede percibir desde la dinámica del proceso, del desarrollo mediato y prolongado de las fuerzas de una sociedad y su interacción con otras, a fin de poder ir determinando con nitidez las fuerzas que dominan su desarrollo. Este concepto, definido como la "Larga Duración" por Braudel en el marco de la Escuela de los Anales, es un instrumento especialmente valioso en el análisis y estudio de las relaciones de nuestro país con sus vecinos del norte, Perú y Bolivia. Lamentablemente, en esos procesos lo que determina muchas veces las decisiones y visiones es, por el contrario, la coyuntura, hecho inmediato y descontextualizado que muchas veces termina convertido en caricatura y fantasía.

Es esta caricatura la que muchas veces pasa a tomar el lugar del estudio desapasionado y de la comprensión del proceso construyendo una visión unidimensional, cuando no ficticia de las contrapartes, hasta llegar a definir un debate en parte relevante irreal. Huelga decir que un proceso semejante se ha desarrollado en Perú y Bolivia, con el agravante de ser los derrotados en la Guerra del Pacífico, lo que ha llevado a una mirada mucho más dura que podemos cristalizar en la trágica frase "cuándo se jodió el Perú", que Mario Vargas Llosa atribuye a su célebre personaje Zavalita en Conversación en la Catedral.

1 Magíster en Ciencia Política, mención Relaciones Internacionales. Profesor de la Facultad de Artes Liberales, Universidad Adolfo Ibáñez. 
De esa forma, la mirada del proceso desaparece ante la mistificación y la proyección de la caricatura, complicando gravemente el establecimiento de una relación saludable.

Una mirada así en Chile ha derivado lentamente en convertir a la relación de Chile con Perú y Bolivia, y especialmente a la Guerra del Pacífico y las demás etapas clave de la relación con Perú y Bolivia, simplemente en hitos rituales de nuestro año cívico. El 21 de mayo y el Combate Naval de Iquique han pasado a ser más relevantes por el ideal ciudadano de la vida y sacrificio de Arturo Prat antes que como una fase relevante de la Campaña Naval de dicho conflicto ${ }^{2}$. Incluso, las operaciones posteriores en Tarapacá, la Campaña de Lima y la Breña posterior han prácticamente desaparecido del devenir diario de nuestra sociedad más allá de ser topónimos urbanos. Nuestra sociedad ha vivido procesos posteriores que han hecho mucho por sacar de la vida cotidiana nuestra posición en la región, y los eventos de encierro posteriores a 1931 sólo agudizaron dicha situación. El abandono, desde 1931, de lo que el profesor Joaquín Fermandois" denominó "nuestra primera mundialización" y la emergencia de la política mesocrática e ideológica nos llevó por otros derroteros en los cuales las consecuencias de una guerra librada hace ya más de 130 años claramente están fuera del interés directo de los que definen la agenda pública.

De esa forma, para la opinión pública chilena es sólo en la condición de coyuntura que aparecen las aspiraciones bolivianas o los reclamos peruanos sobre el límite marítimo. Una elocuente demostración de esta mirada aislada viene dada por la forma coloquial en que el ciudadano chileno se refiere al estado actual de la reclamación peruana por los límites marítimos y su judicialización internacional. Es el "Tema de la Haya", aludiendo a la Corte donde el caso está radicado antes que sus implicancias y complejidades. Es más, no son pocos los que aluden su impaciencia y frustración ante la situación esperando que un fallo, positivo o negativo, concluya "por fin" el

\footnotetext{
2 Sater, William F., La imagen beroica en chile, Arturo Prat, un santo secular. Ediciones Bicentenario, Santiago, 2005.

3 Fermandois, Joaquín, Mundo y fin de mundo; Chile en la política mundial, 1900-2004, Ediciones Universidad Católica de Chile, Santiago, 2005.
} 
problema con el Perú. La caricatura y falta de interés nos lleva a reducir el complejo tema de la relación bilateral sólo al aspecto táctico inmediato, sin mayor interés de comprender su complejidad y profundas raíces históricas. Cuando esa misma persona toma conciencia de que difícilmente las relaciones bilaterales se resolverán de forma mágica con dicho fallo, la esperanza se convierte en frustración, los epítetos aparecen y, finalmente, llega el descarte del tema. Hay cosas más importantes de qué preocuparse.

Ante Bolivia la situación es incluso más aguda, pues no se percibe realmente un "problema" en la aspiración de Bolivia. No es necesario reiterar la afirmación popular en el siglo pasado en que la mejor relación con Bolivia era no tener relaciones. Una mirada que más bien refleja la frustración de no poder resolver algo que ya pasó al plano del imaginario, complicando severamente una solución real.

De esa forma, toda situación es analizada estrictamente desde la perspectiva de la coyuntura, el incidente irrelevante en el largo plazo. No es necesario ser particularmente perspicaz para comprender que un camino así cede toda iniciativa y nos deja a merced de los eventos posteriores, en los que, por ejemplo, la aparición de un tercero con intereses en Bolivia podría de forma sorpresiva alterar drásticamente la situación. Señales de que algo así podría ocurrir en el mediano plazo se han dado de forma sutil pero persistente a la luz del interés brasileño por establecer corredores bioceánicos en el Pacífico.

En el Perú la situación es parecida, aunque no igual. Si bien dicha sociedad ha vivido coyunturas aún más tensionantes de la mano de Sendero Luminoso y, más recientemente, del negocio de la droga con su vínculo con una renacida Guerrilla, la relativa estabilidad de su política interna desde ya hace dos periodos presidenciales hace pensar que el "nunca más" de 1883 adopte ahora formas menos radicales ante Chile. Alan García planteó su campaña presidencial en 2005 sobre la base de "derrotar a Chile en la carrera al desarrollo".

4 Rodríguez Elizondo, José, Temas para después de La Haya. Planeta, Santiago, 2010, p. 129 et seq. 
El fantasma de la recuperación militar fue reemplazado así por una competencia en los indicadores de riesgo y crecimiento. Un avance no menor que, además, se ve fuertemente impulsado desde la perspectiva de la interdependencia compleja de la mano de las elevadas inversiones chilenas en Perú y las crecientes en Chile de parte de capitales peruanos, los que ya han salido del sector terciario para dar sus primeros pasos firmes en la industria mediana. Pese a ello, la frágil institucionalidad peruana, el profundo peso de lo que alguien bautizó como poderes fácticos y la persistencia de profundas asimetrías internas aún encuentran en el discurso anti chileno un refugio de conveniencia. Desde sectores de la elite y burocracia estatales defenestrados por el fujimorato hasta sectores populistas de coyuntura, encuentran siempre el argumento para desenterrar el hacha de guerra ante afrentas reales o pretendidas. Estos grupos pretenden ignorar el -desde su perspectiva inasumible y para nosotros irónicohecho de la profunda coincidencia de la visión de país que las elites han construido en ambos países.

Una mirada similar a la Democracia Liberal, a la Globalización y al comercio abierto como los vehículos para construir sociedades más prósperas ha llegado de forma similar a Santiago y Lima. El que la enorme mayoría de ambas sociedades esté de acuerdo queda nítido con el abrazo al modelo de sociedad de consumo y las aspiraciones sociales verbalizadas en la reciente campaña electoral peruana donde, para triunfar, Ollanta Humala hubo de garantizar la continuidad del modelo una vez que comprendió que la amenaza de la ruptura de este pudo bien haberle costado la elección en el año 2005. Hoy, después de su retórica de visitar Arica en un tanque, se reúne con la elite de inversionistas chilenos en Perú invitándolos a seguir contribuyendo al desarrollo del país. Una mirada apresurada podría considerar esto como una traición a sus ideales. Una mirada más tranquila nos recordará que en América Latina el primer objetivo de un político es retener el poder que ha conseguido y, en el caso de Ollanta Humala, a costa de prender una vela a cada santo. Hoy, cargado con el cumplimiento de esas promesas y con un agravamiento de la narco guerrilla en la zona de los ríos Apurímac y Ene, claramente Chile no figura como adversario militar en el sentido convencional dentro 
del listado de las necesidades reales de dicho gobierno. Al menos así lo demuestra la ausencia sostenida de inversión en el sector Defensa.

En Bolivia la situación es algo diferente. Por un lado el ciclo anímico de la relación vecinal ya ha sido ritualizado desde mediados del siglo pasado en un régimen de esperanza y frustración. Pero esta situación, lejos de permitir una tipificación y solución pragmática, sólo ha servido para lo que el profesor José Rodríguez Elizondo bautizó como la "simplificación", una estructura mecánica convertida en mantra que en los días pasados el ex presidente Paz Zamora resumiera como "querer mar porque lo creemos nuestro"s. Lamentablemente, el Sistema Internacional difícilmente admite dichas simplificaciones en el siglo xxi, más aún cuando el debate se efectúa (¿afortunadamente?) entre Estados que carecen del poder nacional para imponer unilateralmente sus decisiones uno sobre el otro. El problema es que una mirada así, pese a ser inconducente para producir una solución al problema percibido, no por ello deja de existir o ser aceptada por la opinión pública local.

Esta mirada, que explica además de manera algo escapista el fracaso de la elite boliviana en la construcción de Nación y Estado, que sectores cada vez mayores de su sociedad le reprochan, se agudiza en el caso del gobierno del mas. Evo Morales busca romper con una forma de administrar el poder tradicional mostrando éxitos concretos que, a estas alturas, le están resultando cada vez más esquivos. Los incidentes desarrollados durante el año 2011, desde el gasolinazo de febrero hasta la marcha indígena de octubre nos demuestra que la posición de liderazgo e iniciativa que tenía sobre la política boliviana comienza a erosionarse. Como consecuencia relevante de esta situación hay que entender el giro radical desarrollado con ocasión del Día del Litoral el 23 de marzo de 2011, cuando el presidente Morales descartó el proceso de negociaciones en torno a la "Agenda de los 13 Puntos" que había desarrollado con Chile desde la administración Bachelet para entrar en una confusa dinámica de apelaciones internacionales y discursos incendiarios intercalados con llamados al

5 La Segunda, Santiago, Viernes 4 de noviembre, 2011. 
diálogo. La creación de una unidad especial destinada a monitorear el tema marítimo y preparar eventuales recursos a instancias jurídicas internacionales muestra una línea gruesa que busca recurrir a una internacionalización jurídica de la aspiración marítima, y no reclamar a una red de Estados y organizaciones predominantemente regionales que gradualmente han ido perdiendo el entusiasmo o interés en apoyar a una Bolivia que tampoco ha tenido una claridad o persistencia en la estrategia a seguir.

Sólo una cosa sigue constante desde los gobiernos anteriores; la percepción de que Chile "adeuda" a Bolivia no sólo un acceso soberano al océano pacífico, sino que además una suerte de reparación moral y material de las consecuencias que la pérdida del acceso al mar habría supuesto desde 1879. Una suerte de expiación de los pecados que oscila entre el reclamo a un reconocimiento simbólico de responsabilidad a indemnizaciones materiales concretas y que, en algunos casos, han sido catalogadas en cifras astronómicas.

La tragedia es que en este eje se abandonan otros temas también realmente relevantes, desde el comercio bilateral hasta temas fronterizos específicos como los del río Silala o el grave contrabando fronterizo. Bolivia es, al fin y al cabo, el tercer productor de coca en el mundo, y esta es en parte relevante destinada a la producción de narcóticos, los que a su vez tienen que ser exportados a su clientela a través de algún país. Chile crecientemente está reconociendo estas necesidades, pero hasta hace pocos meses parecía ser un tema abandonado. Desde la perspectiva boliviana la situación es aún más grave desde la detención y posterior condena en Estados Unidos del general Sanabria, comandante del esfuerzo antinarcóticos boliviano por narcotráfico.

En un escenario de desencuentros como los descritos, podemos resumir la situación como que cada sociedad ha construido su propio marco de análisis dentro del cual se encuentra cómodamente atrincherado, rechazando cualquier modificación a las matrices en las que ha definido su interés y ámbito de acción. 
El problema es que esta aproximación no sólo resta enormes posibilidades al desarrollo de los tres Estados, sino que además supone riesgos sustanciales de que, ante cualquier cambio brusco del equilibrio de poder, surjan tentaciones coyunturales de "solucionar" el problema de forma unilateral. Es agosto de 1975 y los intentos del septenato peruano Manu Militari, de resolver el tema de Arica son sólo un ejemplo muy nítido del fenómeno ${ }^{6}$.

Ante esta situación el año 2012 nos ofrece un escenario muy interesante. Es cierto, los problemas y desafíos que allí se percibirán serán sustanciales, pero al mismo tiempo de la mano de un fallo en La Haya en esos meses o el primer semestre de 2013 se abrirá un abanico de posibilidades extremadamente rico e interesante.

En primer lugar, claramente habrá riesgos en la definición del fallo y, más allá de quien se percibe finalmente como triunfador o frustrado en sus aspiraciones, abrirá un periodo en que las recriminaciones internas serán poderosas. En Perú se ha construido un escenario maximalista en el que cualquier resultado inferior a un triunfo absoluto será percibido con frustración y molestia, situación incómoda para un presidente como Ollanta Humala, quien hizo su carrera política inicial basándose en un proceso con una fuerte carga de hostilidad vecinal. Por su lado, para un Chile que se dejó imponer una estructura de reclamación en la que no tuvo mayor iniciativa, cualquier solución que no respete su soberanía integral en el territorio marítimo actualmente controlado será percibido como un detrimento a su patrimonio territorial y una derrota concreta y factual.

Peor aún es difícil encontrar en fallos de la Corte de La Haya resoluciones radicales que favorezcan de forma absoluta a un litigante en detrimento del otro, por lo que es una posibilidad seria de que ambos litigantes queden frustrados, generando una situación "LoseLose" que constituirá un complejo desafío para ambas cancillerías y, sobre todo, para los líderes de opinión de ambos países. Canalizar

6 Velaochaga, Andrés, Historia diplomática del Perú, Editorial Universidad Martín de Porres, Lima, 1997. 
la frustración no va a ser un tema menor. Afortunadamente, no parece existir el interés ni la capacidad por recurrir a instrumentos o aproximaciones de fuerza, aunque sólo sea porque la guerra se ha vuelto un recurso políticamente muy caro en el siglo xxi. Nadie puede arriesgarse a perder posiciones ante las clasificadoras de riesgo y decisores de inversión. Lo que sí no es descartable desde el lado peruano es un eventual estallido interno de sectores radicalizados que pudieran recurrir a algún grado de violencia, mientras que en Chile el debate por la asignación de responsabilidades difícilmente sería tranquilo y mesurado viendo el grado de crispación y desorientación de la política interna actual.

Para el caso boliviano, si bien no directamente involucrado, especialmente tras el descarte en la Corte del extraño documento presentado ante ella reclamando derechos "expectaticios" ante el resultado final del fallo, no es menor considerar que la definición del límite marítimo es clave en la materialidad de un acceso boliviano en Arica. Y es que un corredor en el eje propuesto en Charaña es no sólo la única alternativa realmente viable de conexión entre el Hinterland andino de Bolivia con el Pacífico, sino además la tradicional e histórica vía de egreso de la minería del Altiplano al Pacífico desde tiempos coloniales. No es casualidad que Francis Drake capturara un galeón cargado de Plata en Arica en $1580^{7}$.

Para Bolivia, el Tratado de 1929 ya complicó gravemente cualquier opción de acceso al mar por el área pero, una modificación del statu quo a través de cualquier medida que incremente las facultades peruanas en el área sólo agravaría esta situación. De ahí la persistente frustración de Evo Morales ante el litigio chileno-peruano, que lo ha llevado en más de alguna ocasión a calificar la presentación limeña como un agresión directa a las pretensiones bolivianas ${ }^{8}$.

\footnotetext{
7 Bawlf, Samuel, The secret voyage of Sir Francis Drake, 1577-1580, Penguin Books, Londres, 2004.

8 "La demanda [de García] en La Haya es para mejorar su imagen y perjudicar a Bolivia [...] También tengo la información de que el Gobierno peruano sabe que la demanda la va a perder", En http://gestion.pe/noticia/294154/morales-peru-sabe-que-va-perder-demanda-chile, revisado el 24 de octubre de 2011.
} 
En un escenario así, con el gobierno del mas muy debilitado y ante la necesidad de producir resultados concretos, la situación se vuelve especialmente grave en términos de que todos los actores pueden percibir tener graves intereses en riesgo.

Pero, y este es el quid del asunto en nuestra opinión, esta es a la vez la oportunidad de poder definir un primer paso en el camino rumbo a la solución. Y quizás este es el paso más relevante, pero el que menos se ha definido con anterioridad. La coyuntura ha llevado a los tres actores a considerar que la solución es sinónimo o equivalente a su solución, la única aceptable en un esquema de suma 0 . Pero en un escenario de riesgo perfectamente puede existir el interés de reordenar el escenario, de limitar eventuales pérdidas y de buscar una solución transaccional. Existe una opción de flexibilizar las miradas específicas y comenzar a abrirse a visiones de más trascendencia, donde el otro ocupe un lugar en la construcción de una mirada aceptable.

En ese sentido quizás la primera tarea es definir qué solución se busca, es decir, definir los mínimos absolutos de cada posición y su interrelación. No necesitamos plantear lo difícil y complejo que resulta realizar esto, pero en un marco razonable de negociación y confianza, así como de interés propio, no es imposible pensar que podría construirse un escenario así.

Necesariamente, una mirada de este estilo requiere un análisis de compatibilidades que primero establezca las bases de confianza chileno-peruanas respecto al límite marítimo y a la posición del hito número $1 \mathrm{y}$ del punto concordia. Una mirada clara en estos aspectos, permite definir luego el interés (o ausencia de este, no es posible negarlo como alternativa) de ambas partes en resolver el tema de la aspiración boliviana. Esta situación bilateral puede por tanto convertirse en trilateral una vez que tenga los cimientos para poder debatir una estructura de estas características. Esta trilateralización, sin embargo, tiene que tener claramente definidos sus límites para no convertirse en una caja de Pandora que termine siendo peor que 
la situación de base, y colapsar como las conversaciones de Charaña ante las intenciones de invasión de Juan Velasco Alvarado.

Una buena forma de canalizar dicha situación es la construcción de redes de interdependencia compleja a nivel de líderes y creadores de opinión. No nos referimos sólo a la coyuntura y a los medios de comunicación, sino más bien a la academia y a las sociedades civiles respectivas9. Fomentar un intercambio sostenido y fructífero necesariamente será un proceso largo, pero esto tiene que comenzar algún día si es que realmente se pretende llegar a un modus vivendi efectivo. Obviamente habrá tropiezos y frustraciones, pero en la lenta construcción de confianzas está la llave para que la solución eventual pueda tener base política y no siga la suerte de tantas soluciones de gabinete con pretensiones de secreto, es decir, quedar reducidas al anecdotario diplomático o político.

Y es que este es el núcleo de la situación. Una solución al tema de las fronteras del norte de nuestro país y a las relaciones con Bolivia y Perú necesariamente requiere de la construcción de una base de confianzas que tiene que construirse en la larga duración. No se puede pretender producir soluciones mágicas que, como conejo sacado de una chistera, resuelvan de un solo golpe las situaciones que se han arrastrado ya desde la Independencia o poco después. Es crítico por tanto reconocer que el año 2012 casi con seguridad no será el año de la "solución" sea cual sea, pero bien puede ser el año en que se comience a construir el PROCESO para llegar a unas relaciones menos crispadas y que satisfagan los mínimos indispensables para cada actor del complejo cuadrante sudoriental del Pacífico. Sólo una vez conseguido eso se podrá pasar a los temas de fondo.

9 En este sentido resulta encomiable el esfuerzo conjunto realizado por los profesores Cristóbal Aljovin y Eduardo Cavieres en relación a una aproximación conjunta a la Guerra del Pacífico. 